\title{
Paramedian thalamic stroke syndrome
}

\author{
Z I Lockhat, FFRad (D)SA \\ S Raven, MB ChB \\ Department of Radiology, Pretoria Academic Hospital, University of Pretoria

\section{$M$ van Rensburg, MB ChB, MMed Rad (D), FRCR, FRANZCR} \\ Private Practice, Wilgers MR, Pretoria \\ I van de Werke, FRCR \\ Department of Radiology, Kalafong Hospital, University of Pretoria
}

\section{Introduction}

Bilateral paramedian thalamic infarction is rare. The suggested mechanism is occlusion of a single central thalamic perforating artery - an anatomic variant described by Percheron in 1977. ${ }^{1}$ Bilateral paramedian thalamic infarctions may result in the classic triad of hypersomnolence (excessive sleepiness), ocular disturbances (supranuclear and vertical gaze palsy) and neuropsychological deficits (including an amnestic syndrome). ${ }^{2}$ The paramedian thalamus is believed to play an important role in the regulation of sleep and disturbances in sleep regulation are known to occur in paramedian thalamic stroke (PTS).

Because of the absence of lateralising signs in PTS ('inobvious stroke') disturbance of consciousness may initially be assumed to be of metabolic-toxic origin. However the triad of hypersomnia, neuropsychological deficits (frontal syndrome and amnesia) and eye-movement disturbance allows the recognition of the vascular syndrome. Vertical gaze palsy is the best clinical predictor of the degree of hypersomnia, whereas severe amnesia can also occur in patients with mild hypersomnia. In mildly affected patients, hypersomnia usually resolves within a few months, whereas in severe cases a disabling hypersomnia may persist for $>1$ year. Eventually hypersomnia resolves, leaving some patients in a state of apathy, psychomotor slowing, intentional deficits and amnesia that has been called 'thalamic dementia'.

The word thalamus (from the Greek thalamos), refers to an inner room or bridal chamber therefore the posterior part of the thalamus is appropriately named 'pulvinar' (pillow in Greek). ${ }^{2}$ The thalami contain strategic nuclei and integrate several important cortical functions. These nuclei are involved with memory, emotion and maintenance of consciousness. Thus infarcts at the mesencephalic diencephalic junctions may result in complex clinical syndromes with a wide range of symptoms varying from motor deficits to behavioural and sensory alterations.

Thalamic infarctions result in markedly different clinical syndromes on the basis of the location of the infarction in relation to the thalamic nuclei involved. The location of the infarction is determined by the blood supply distribution to the thalamus and midbrain. Hence an understanding of the vascular supply to the thalami is highlighted. The arterial supply to these areas arises from many perforating blood ves- sels with a complex distribution for which many variations have been described. One rare variation, named the 'artery of Percheron' is a solitary arterial trunk that arises from one of the proximal segments of a posterior cerebral artery and supplies the paramedian thalami and the rostral midbrain bilaterally. ${ }^{2}$

\section{Imaging findings}

A 48-year-old man presented with hypersomnia 5 days after stopping antihypertensive therapy. On examination he was very sleepy with vertical gaze paresis and short-term memory loss. MRI demonstrated high signal intensity on T2 and FLAIR in the thalami bilaterally (low T1 signal) which was positive on diffusion-weighted images (Figs 1 - 4).

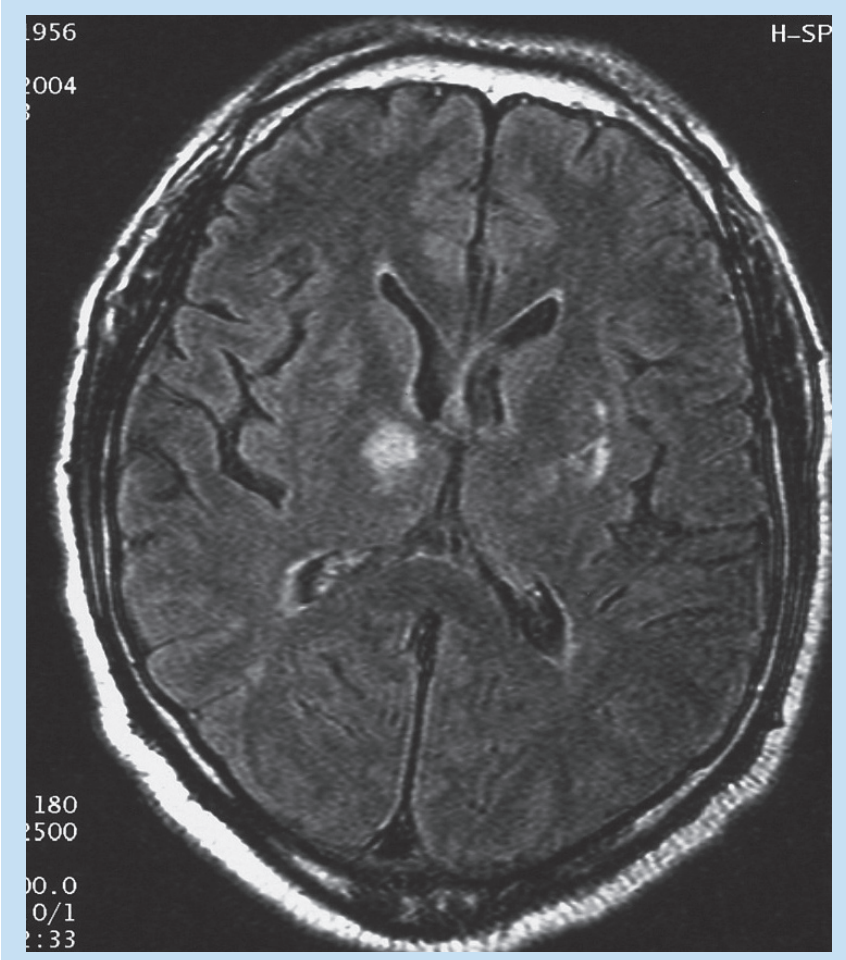

Fig. 1. Axial T2-weighted flair image shows high intensity lesions in thalami bilaterally.

\section{Discussion}

The thalamus is believed to play a role in the regulation of sleep. Since the report of Freund in 1913 PTS has been known to be associated with hypersomnia. Previously hypersomnia associated with PTS has been attributed to diminished arousal signals ascending from the brain stem, however this is challenged by the suggestion that the thalamus also plays an important part not only in maintaining the wakeful state but also in producing sleep.

The hypersomnia seems to arise from the simultaneous impairment of mechanisms for maintenance of arousal and for promotion of nonREM sleep, both of which depend on the integrity of the paramedian 


\section{SHORT REPORT}

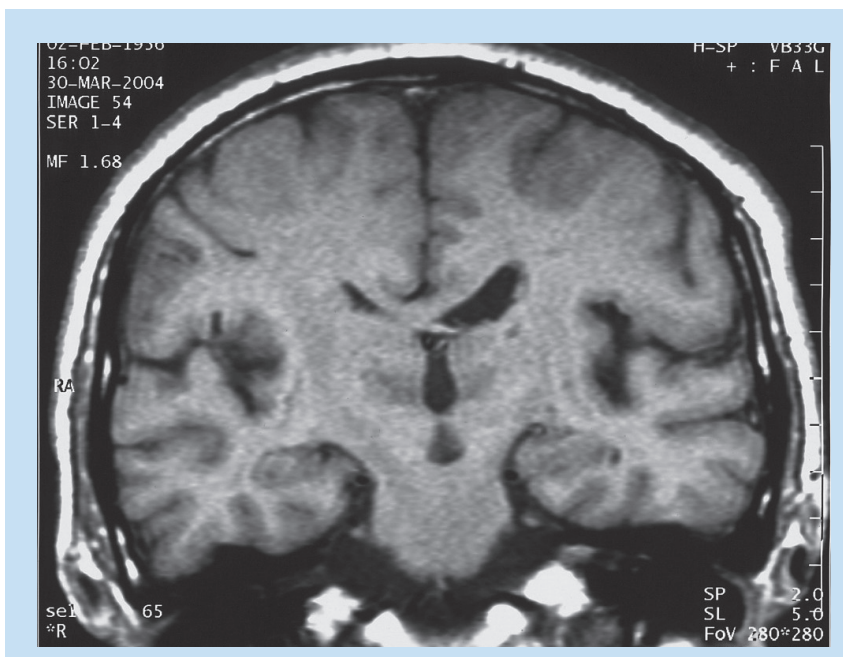

Fig. 2. Coronal T1-weighted image with bilateral low signal intensities in the thalami.

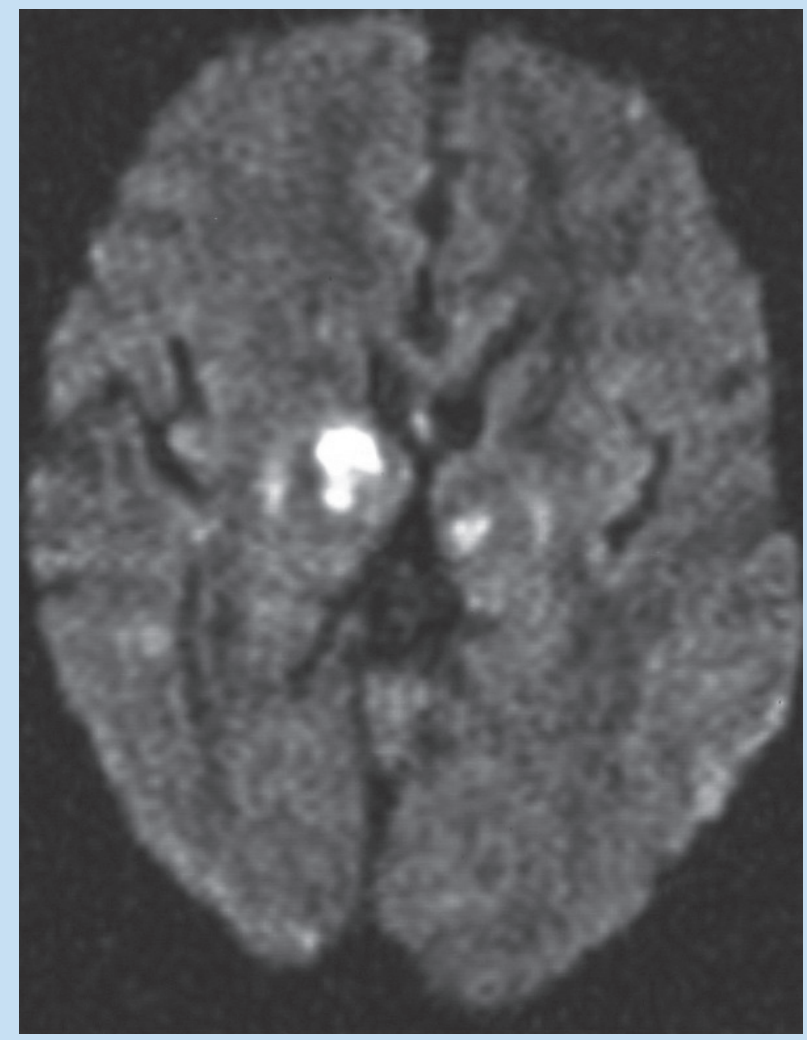

Fig. 3. Axial diffusion-weighted image with bilateral high signal intensity in the thalami.

thalamus. ${ }^{2}$ The thalamus is vascularised by branches of four arterial pedicles that arise from the basilar artery bifurcation, the posterior communicating and posterior cerebral arteries. The paramedian aspect of the thalamus is supplied by the thalamic-subthalamic artery.

Four main thalamic vascular territories are recognised: $(i)$ the territory supplied by the infero-lateral arteries, i.e. the ventral posterior group

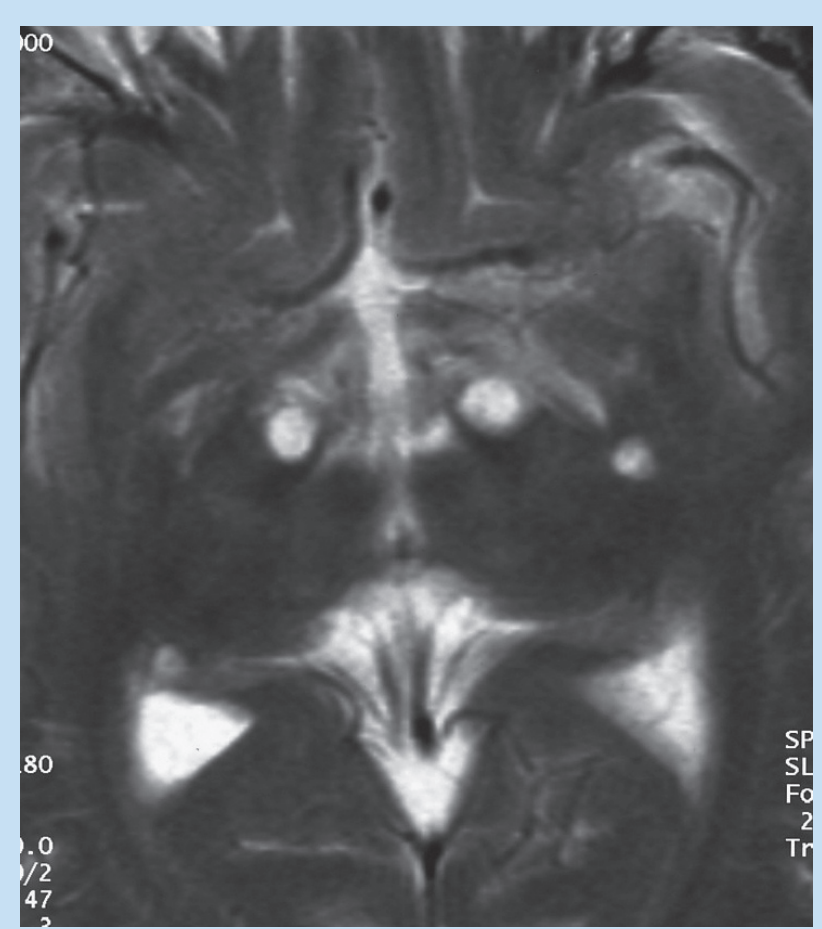

Fig. 4. Axial T2-weighted image with bilateral high signal intensity in the thalami.

nuclei; (ii) the territory of the posterior choroidal arteries corresponding to the anterior region including the ventral anterior nucleus and the ventral lateral nucleus; (iii) the territory of the posterior choroidal arteries, supplying the lateral geniculate body; and (iv) the paramedian territory, supplying the medial thalamic nuclei.

Consequently, thalamic infarcts are classified anatomically into four distinct syndromes: the inferolateral, tuberothalamic, posterior choroidal, and paramedian stroke syndrome. The most frequent type of infarction corresponds to the inferolateral territory. The paramedian aspect of the thalamus is supplied by one or several paramedian thalamic arteries. $^{2}$

Percheron demonstrated that both paramedian thalami are vascularised by a single common vessel in approximately one-third of cases. Occlusion of this vessel gives rise to bilateral lesions.

Occasionally a common vascular pedicle supplies both the paramedian thalamic arteries, and the paramedian mesencephalic arteries (also called posterior thalamo-subthalamic artery). Such common origin explains the occurrence of paramedian infarction extending from the thalamus to the upper midbrain ${ }^{2}$ (Fig. 5).

The aetiology of thalamic strokes may vary; they can be due to lacunar infarction, large artery atherosclerosis, with artery-to-artery embolism, cardioembolism, deep venous thrombus and migrainous stroke. ${ }^{4}$ Bilateral thalamic lesions can be caused by several other conditions such as thiamine deficiency, cerebral lupus, toxoplasmosis, cysticercosis, syphilis, tumours and fungal infections.

Patients with unilateral infarcts of the area supplied by the paramedian thalamic-subthalamic arteries frequently have a classic diencephalic triad of an acutely decreased level of consciousness, neuropsychological 


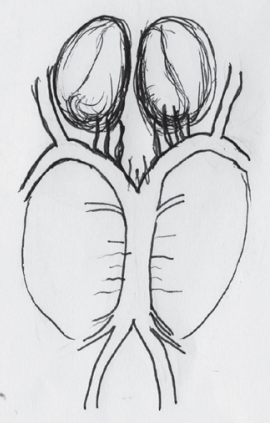

A

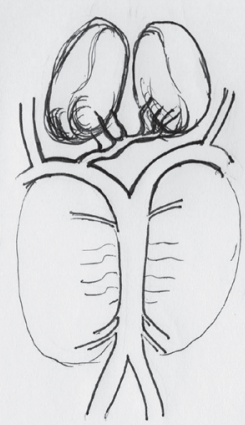

B

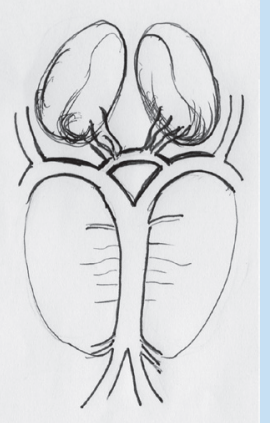

C
Fig. 5. Schematic diagram. (A). Multiple small perforating arteries arising from the $P 1$ segments of both posterior communicating arteries (most common). (B). A single perforating artery arising from only one of the $P 1$ segments (the artery of Percheron). (C) An arcade of perforating arteries arising from a bridging artery between the $P 1$ segments of the posterior communicating arteries.

disturbances and vertical gaze palsy. The severity of disturbance of vigilance may range from simple lethargy to hypersomnia and coma, depending upon the extent of subthalamic involvement.

\section{Conclusion}

From a practical standpoint bilateral paramedian thalamic infarcts should be recognised as due to occlusion of a possible single rare artery that is a normal anatomic variant. This should not be attributed to occlusion of multiple vascular territories or other pathologic conditions such as vasculitis or infectious disease.

Supratentorial bilateral symmetrical arterial distributions are unusual. Occlusion of an azygous anterior cerebral artery may result in bilateral infarction. When bilateral medial thalamic infarcts are encountered the differential diagnosis should also include the top of the basilar artery syndrome. In this latter entity infarctions tend to involve also the territories supplied by the superior cerebellar and posterior cerebral arteries.

When bilateral paramedian thalamic infarcts are found, occlusion of the artery of Percheron should be considered as the main diagnosis.

Raitberg B, Tuccar E, Alp M. Bilateral paramedian thalamic infarct in the presence of an unpaired thalamic perforating artery. Acta Neuro Chir (Wien) 2002; 144: 301 - 304.

2. Lövblad KO, Basetti C, Remanda L, Schroth G. Paramedian thalamic stroke syndrome. Int J Neurol 1997 8: 116 - 122.

3. Basetti C, Mathis J, Gugger M, Lövblad KO, Hess C. Hypersomnia following paramedian thalamic stroke: a report of 12 patients. Ann Neurol 1996; 39: 471 - 480.

4. Matheus G, Castillo M. Imaging of acute bilateral paramedian thalamic and mesencephalic infarcts. Am J Neuroradiol 2003; 24: 2005 - 2008.

\section{ESSENTIAL PATHOLOGY THIRD EDITION}

\section{Member: R440.00 Non Member: R470.00}

To order contact Carmen or Avril: Tel: (02 1) 530-6520/27 Fax: (021) 531-4126/3539

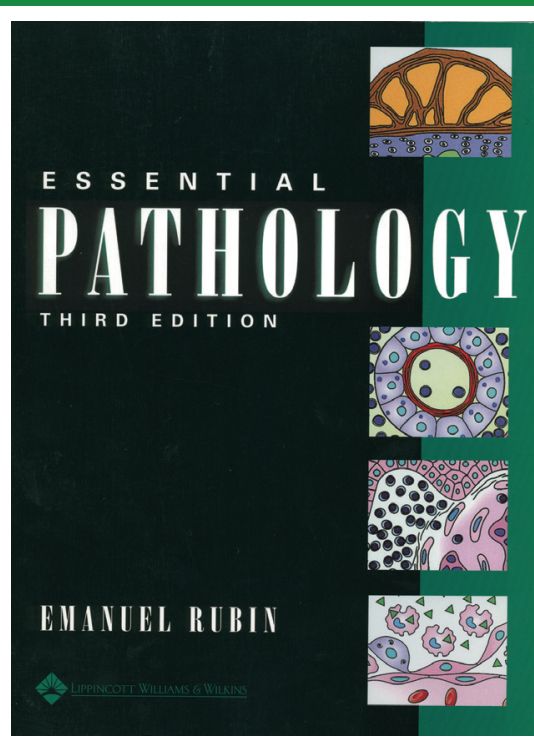
email: carmena@hmpg.co.za or avrilm@hmpg.co.za HMPG

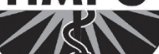

Health \& Medical Books

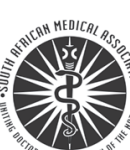

The South African Medical Association, Health \& Medical Publishing Group, Suites 1-2 Lonsdale Building, Gardener Way, Pinelands, 7405. 